\title{
A Practical Synthetic Route to Enantiopure 6-Substituted Cis-Decahydroquinolines ${ }^{\dagger}$
}

\author{
Mercedes Amat, ${ }^{\star}$, Laura Navío, ${ }^{\ddagger}$ Núria Llor, ${ }^{\ddagger}$ Elies Molins,${ }^{\S}$ and \\ Joan Bosch ${ }^{\ddagger}$
}

Laboratory of Organic Chemistry, Faculty of Pharmacy, and Institute of Biomedicine (IBUB), University of Barcelona, 08028-Barcelona, Spain, and Institut de Ciència de Materials de Barcelona (CSIC), Campus UAB, 08193-Cerdanyola, Spain

amat@ub.edu

Received Date (will be automatically inserted after manuscript is accepted)

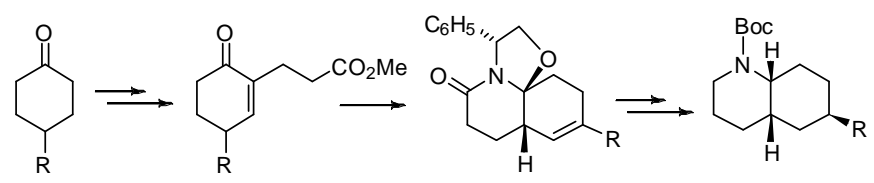

Starting from 4-substituted cyclohexanones, a practical synthetic route to enantiopure 6-substituted cisdecahydroquinolines has been developed, the key steps being a stereoselective cyclocondensation of an unsaturated $\delta$-keto ester derivative with $(R)$-phenylglycinol and the stereoselective hydrogenation of the resulting tricyclic oxazoloquinolone lactams.

Bicyclic phenylglycinol-derived oxazolopiperidone lactams provide a general solution for the synthesis of enantiopure polysubstituted piperidines bearing virtually any type of substitution pattern, including indolizidines, quinolizidines, hydroisoquinolines, other fused and bridged piperidine derivatives, and more complex piperidine-containing natural products and bioactive compounds. ${ }^{1}$

Using related tricyclic oxazoloquinolone lactams as enantiomeric scaffolds, we have recently developed a procedure that allows easy access to enantiopure 5substituted decahydroquinolines. ${ }^{2}$ Apart from their

${ }^{\dagger}$ Dedicated to Prof. Francisco Palacios on the occasion of his $60^{\text {th }}$ birthday.

University of Barcelona

$\S$ Institut de Ciència de Materials

${ }^{1}$ For recent reviews, see: (a) Amat, M.; Pérez, M.; Bosch, J. Synlett 2011, 143-160. (b) Amat, M.; Llor, N.; Griera, R.; Pérez, M.; Bosch, J. Nat. Prod. Commun. 2011, 6, 515-526. (c) Amat, M.; Pérez, M.; Bosch, J. Chem. Eur. J. 2011, 17, 7724-7732. For pionering work in the field, see: (d) Brengel, G. P.; Meyers, A. I. Chem. Commun. 1997, 1-8. (e) Groaning, M. D.; Meyers, A. I. Tetrahedron 2000, 56, 98439873.

Amat, M.; Fabregat, R.; Griera, R.; Florindo, P.; Molins, E.; Bosch, J. J. Org. Chem. 2010, 75, 3797-3805. interest as bioactive compounds, ${ }^{3}$ decahydroquinolines bearing substituents at the carbocyclic ring are very attractive synthetic targets as there are few methodologies for their enantioselective synthesis, ${ }^{4}$ with no precedents for the preparation of 6-substituted derivatives.

In this letter, we disclose a practical synthetic route to enantiopure 6-substituted cis-decahydroquinolines using 4-substituted cyclohexanones $\mathbf{1}$ as the starting materials. The key steps of the synthesis are a stereoselective cyclocondensation of $(R)$-phenylglycinol with an

3 (a) Daly, J. W. In The Alkaloids; Cordell, G. A. Ed.; Academic Press: New York, 1998; Vol. 50, pp 141-169. (b) Daly, J. W.; Garraffo, H. M.; Spande, T. F. In Alkaloids: Chemical and Biological Perspectives; Pelletier, S. W., Ed.; Pergamon, New York, 1999; Vol. 13, pp 1- 161. (c) Spande, T. F.; Jain, P.; Garraffo, H. M.; Pannell, L. K.; Yeh, H. J. C.; Daly, J. W. Fukumoto, S.; Inamura, K.; Tokuyama, T.; Torres, J. A.; Snelling, R. R.; Jones, T. H. J. Nat. Prod. 1999, 62, 5- 21. (d) Daly, J. W.; Spande, T. F.; Garraffo, H. M. J. Nat. Prod. 2005, 68, 1556-1575. (e) For a review on the synthesis of decahydroquinolines, see: Kibayashi, C.; Aoyagi, S. In Studies in Natural Products Chemistry; Atta-ur-Rahman, Ed.; Elsevier, Amsterdam, 1997; Vol. 19, pp 3-88.

${ }^{4}$ (a) Heitbaum, M.; Fröhlich, R.; Glorius, F. Adv. Synth. Catal. 2010, 352, 357-362. (b) Pham, V. C.; Jossang, A.; Grellier, P.; Sévenet, T.; Nguyen, V. H.; Bodo, B. J. Org. Chem. 2008, 73, 75657573. 
unsaturated $\delta$-keto ester derivative $\mathbf{3}$ and the stereoselective carbon-carbon double bond hydrogenation of the resulting tricyclic lactam 4 , taking advantage of the conformational rigidity of the tricyclic system.

The required cyclohexenone esters 3 were prepared from cyclohexanones $\mathbf{1}$ as outlined in Scheme 1, either via bromination-elimination of $\delta$-keto esters $\mathbf{2}$ (series $\mathbf{a}, \mathbf{b}$; $55-60 \%$ overall yield) or by alkylation of a keto sulfoxide ${ }^{5}$ intermediate with methyl acrylate, followed by thermal elimination (series $\mathbf{c}-\mathbf{e} ; \sim 75 \%$ overall yield).

Scheme 1. Preparation of the Starting Unsaturated Keto Esters

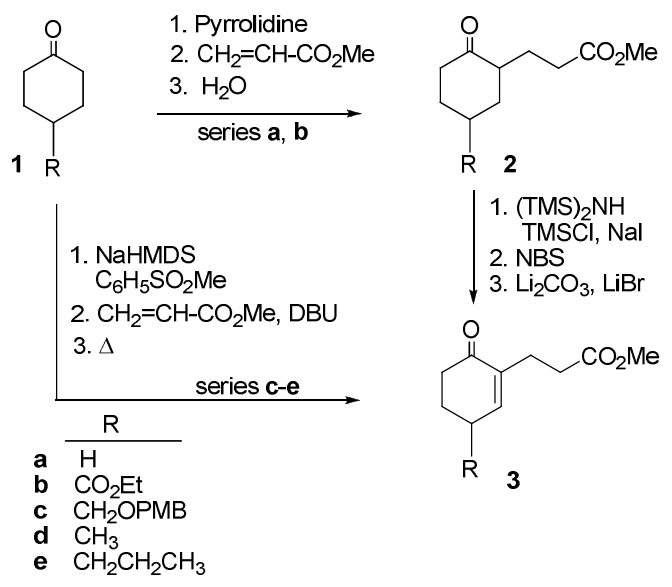

Treatment of unsaturated keto esters 3b-e with $(R)$ phenylglycinol in a Dean-Stark apparatus, in refluxing toluene containing isobutyric acid, stereoselectively led to tricyclic cis-hydroquinoline lactams 4 , in which the migration of the carbon-carbon double bond has occurred (Scheme 2). Minor amounts of the cis-diastereoisomers 5 $(7 \mathrm{a} R, 11 \mathrm{a} R)$ were also formed (approximate $4: 1$ ratio; 75 $80 \%$ overall yield).

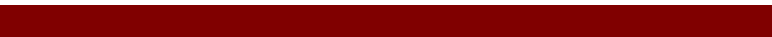

Scheme 2. Cyclocondensation Reactions with (R)Phenylglycinol

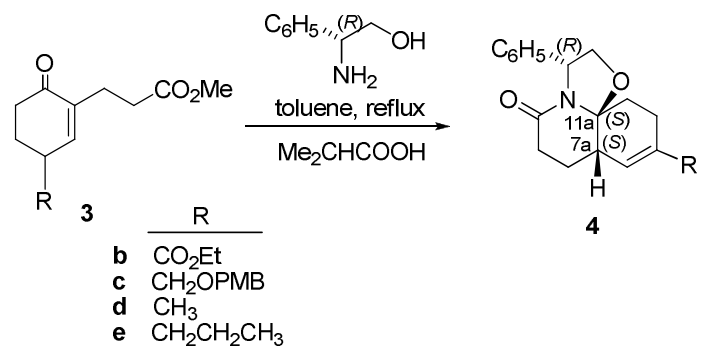

The formation of these lactams can be accounted for by considering that the initially formed conjugated imines $\mathbf{A}$

\footnotetext{
${ }^{5}$ Monteiro, H. J.; De Souza, J. P. Tetrahedron Lett. 1975, 921-924.
}

are in equilibrium, via dienamines $\mathbf{B}$, with two epimeric $\beta, \gamma$-unsaturated imines $\mathbf{C}$ and four diastereoisomeric oxazolidines $\mathbf{D}$, as outlined in Scheme 3.

Scheme 3. Mechanistic Pathway for the Cyclocondensation Reaction

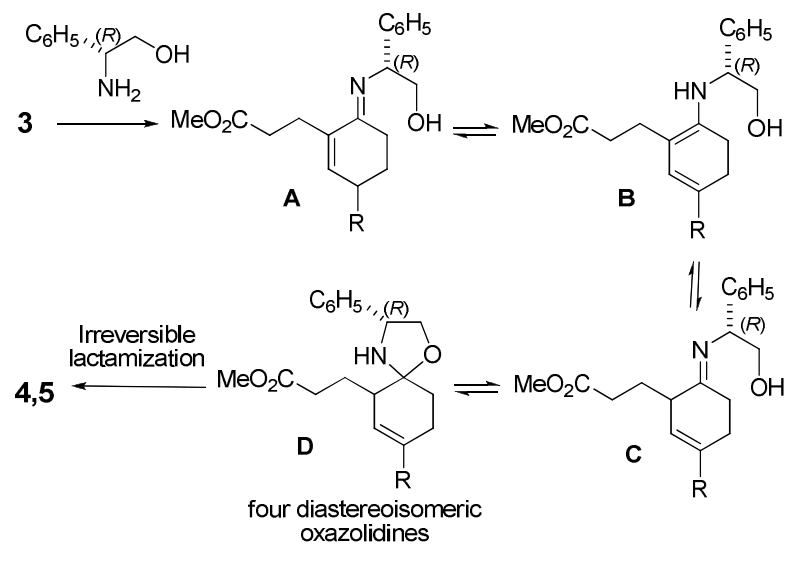

Due to steric constraints, the subsequent irreversible lactamization occurs only from the diastereoisomers ox-1 and ox-2 that lead to the cis fused hydroquinolones $\mathbf{4}$

Scheme 4. The Lactamization Step
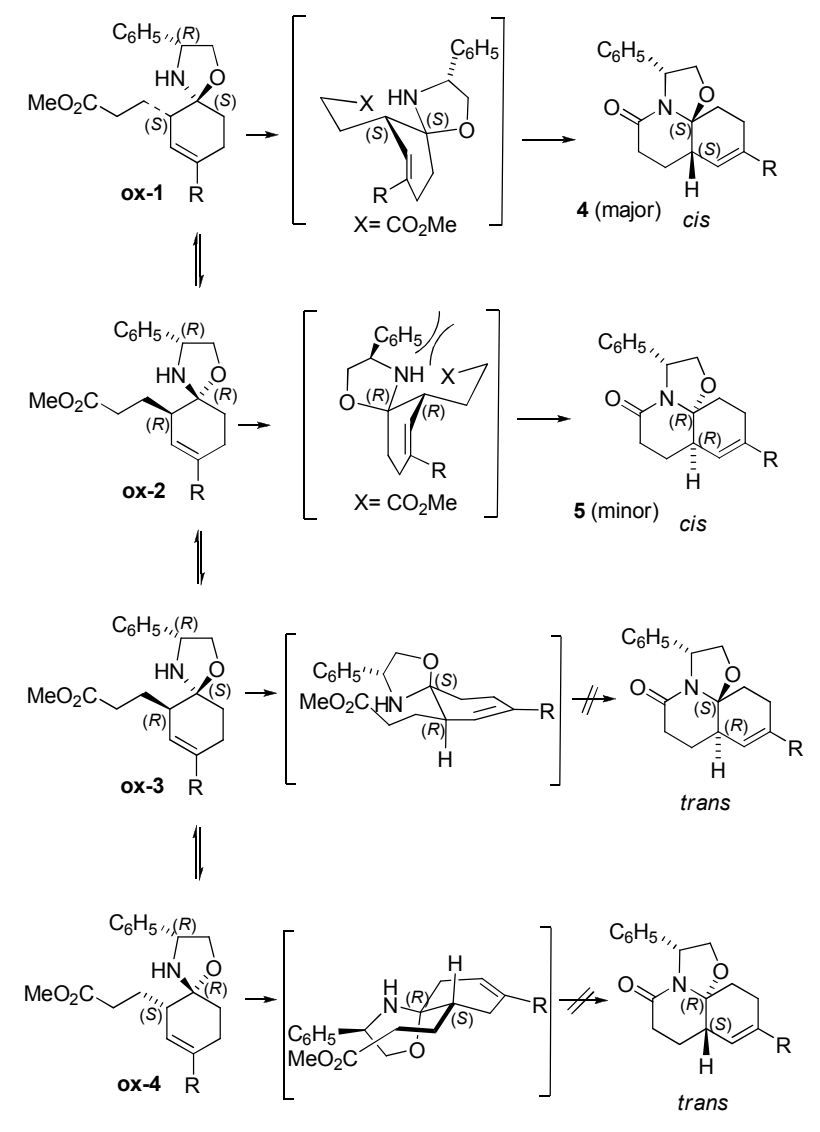
(major) and $\mathbf{5}$ (minor), via a chair-like transition state in which the unsaturated carbon moiety of the cyclohexene ring adopts an equatorial disposition with respect to the incipient six-membered lactam ring (Scheme 4). The cyclization occurs faster from ox-1, and consequently tricyclic lactam $\mathbf{4}$ is the major product of the cyclocondensation reaction, as this oxazolidine allows a less hindered approach of the ester group to the nitrogen atom, avoiding the repulsive interaction with the phenyl substituent. ${ }^{6}$ No lactams with a trans hydroquinoline ring fusion were observed.

Catalytic hydrogenation of lactams $4 \mathbf{b}, \mathbf{d}, \mathbf{e}$ in $\mathrm{MeOH}$ using $\mathrm{PtO}_{2}$ as the catalyst took place in excellent yield with high facial selectivity, with uptake of hydrogen from by the most accessible $\alpha$-face to give the respective decahydroquinolines 6 (Scheme 5). Minor amounts of the corresponding C-9 epimers were also formed.

An X-ray crystallographic analysis of lactam $\mathbf{6 b}$ unambiguously confirmed the absolute configuration of the new stereogenic center generated in the hydrogenation step and of the hydroquinoline ring junction carbons formed in the cyclocondensation reaction.

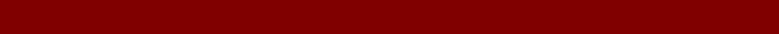
Scheme 5. Synthesis of Enantiopure 6-Substituted Cis-Decahydroquinolines

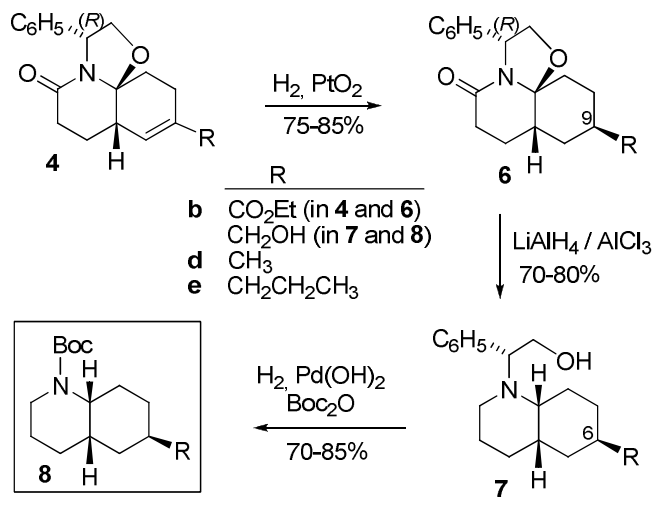

Alane reduction of crude tricyclic lactams 6 brought about the stereoselective ${ }^{7}$ reductive opening of the oxazolidine ring and the reduction of the lactam and ester (in series b) carbonyl groups to give cisdecahydroquinolines $\quad 7^{8} \quad \mathrm{~A}$ subsequent catalytic debenzylation in the presence of $\mathrm{Boc}_{2} \mathrm{O}$ led to 6substituted decahydroquinolines 8. Taking into account the availability of the starting 4-substituted

${ }^{6}$ For the stereochemical outcome of related cyclocondensation reactions from $\delta$-keto esters, see: (a) Amat, M.; Cantó, M.; Llor, N.; Escolano, C.; Molins, E.; Espinosa, E.; Bosch, J. J. Org. Chem. 2002, 67, 5343-5351. (b) Amat, M.; Bassas, O.; Llor, N.; Cantó, M.; Pérez, M.; Molins, E.; Bosch, J. Chem. Eur. J. 2006, 12, 7872-7881.

Fréville, S.; Célérier, J. O.; Thuy, V. M.; Lhommet, G. Tetrahedron Asymmetry 1995, 6, 2651-2654. See also ref 6a.

8 At this stage, minor amounts of 6-epi-7d and 6-epi-7e, formed from the minor epimers generated in the hydrogenation step, were isolated. cyclohexanones, the sequence reported here provides a general route to enantiopure 6-substituted cisdecahydroquinolines.

Similar cyclocondensation reactions of unsaturated keto esters 3a-c and the saturated keto ester $3 \mathbf{f}$ with $(S)$ tryptophanol $^{9} \quad$ (Scheme 6) were also highly stereoselective, leading to the corresponding cis lactams 9 $(3 S, 7 \mathrm{a} R, 11 \mathrm{a} R)$ as the major products [the ratio 9:( $3 S, 7 \mathrm{a} S, 11 \mathrm{a} S)$-isomers was $4: 1 ; 65 \%-75 \%$ overall yield]. This significantly expands the potential of tricyclic oxazoloquinolone lactams as chiral building blocks since $(S)$-tryptophanol not only acts as a chiral inductor in the cyclocondensation reaction, which was the role of $(R)$ phenylglycinol, but can also be used to assemble more complex hydroquinoline-fused derivatives. Thus, Bischler-Napieralski cyclization of tricyclic lactams 9a,c, $\mathbf{f}^{10}$ followed by $\mathrm{LiAlH}_{4}$ reduction of the resulting allcis hexacyclic derivatives $\mathbf{1 0}$ stereoselectively led in excellent yield (85-90\% overall yield) to pentacyclic amino alcohols 11, which embody the pentacyclic skeleton of tangutorine. ${ }^{11}$

Scheme 6. Cyclocondensation Reactions with $(S)$-Tryptophanol

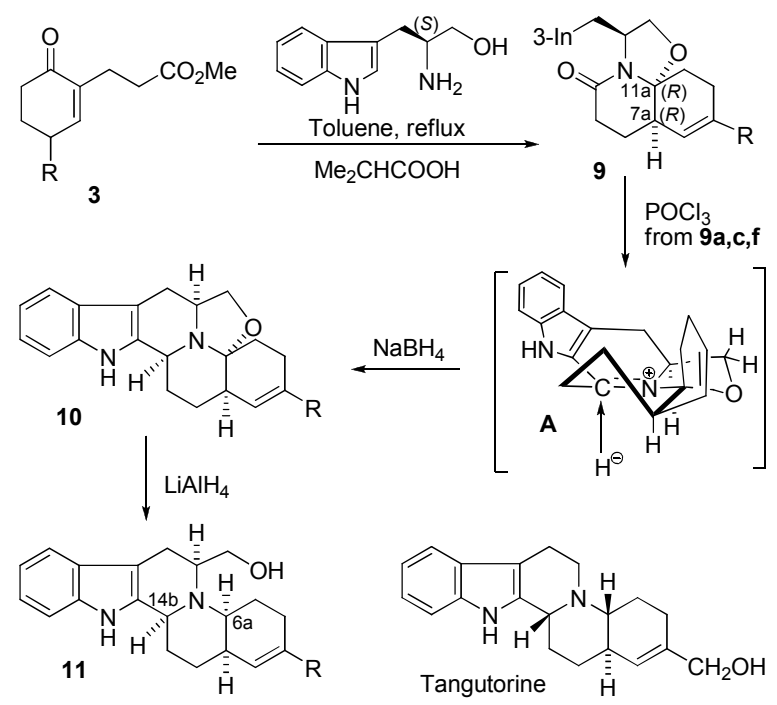

a R= H; b R= $\mathrm{CO}_{2} \mathrm{Et} ; \mathbf{c} \mathrm{R}=\mathrm{CH}_{2} \mathrm{OPMB}\left(\mathrm{CH}_{3}\right.$ in 11); $\mathbf{f} \mathrm{R}=\mathrm{H}$ (dihydro) 3-In= 3-Indolyl

\footnotetext{
${ }^{9}$ For cyclocondensation reactions of $\delta$-oxo acid derivatives with $(S)$ tryptophanol, see: (a) Allin, S. M.; Thomas, C. I.; Doyle, K.; Elsegood, M. R. J. J. Org. Chem. 2005, 70, 357-359. (b) Amat, M.; Santos, M. M. M.; Bassas, O.; Llor, N.; Escolano, C.; Gómez-Esqué, A.; Molins, E.; Allin, S. M.; McKee, V.; Bosch, J. J. Org. Chem. 2007, 72, 51935201. (c) Amat, M.; Gómez-Esqué, A.; Escolano, C.; Santos, M. M. M.; Molins, E.; Bosch, J. J. Org. Chem. 2009, 74, 1205-1211. (d) Allin, S. M.; Duffy, L. J.; Towler, J. M. R.; Page, P. C. B.; Elsegood, M. R. J., Saha, B. Tetrahedron 2009, 65, 10230-10234.

10 Under classical conditions $\left(\mathrm{POCl}_{3}\right.$, then $\left.\mathrm{NaBH}_{4}\right)$ attempted Bischler-Napieralski cyclization from $(S)$-tryptophanol-derived lactams lacking the substituent at the aminal carbon resulted in failure due to the tendency of these lactams to undergo $\alpha$-amidoalkylation under acidic condicitions: see ref $9 \mathrm{c}$.

${ }^{11}$ Duan, J.-A.; Williams, I. D.; Che, C.-T.; Zhou, R.-H.; Zhao, S.-X. Tetrahedron Lett. 1999, 40, 2593-2596.
} 
The configuration of the two stereogenic centers generated in the cyclocondensation reaction was unambiguously established by X-ray diffraction analysis of the thiolactam derived from $9 \mathbf{a}$, which was prepared in $77 \%$ yield by treatment of $9 \mathbf{a}$ with Lawesson's reagent. On the other hand, the configuration of the C-6a and C$14 \mathrm{~b}$ stereocenters of $\mathbf{1 1}$ was deduced from the NMR data (COSY, HETCOR and NOESY experiments), by considering a preferred cis-cisoid-cis conformation, ${ }^{12}$ and by comparison of the ${ }^{13} \mathrm{C}$ NMR chemical shifts with the values reported for tangutorine ${ }^{11}$ (see Supporting Information).

The stereoselectivity of the Bischer-Napieralski cyclization can be rationalized by considering that the attack of the hydride on the electrophilic carbon center of the conformationally rigid iminium intermediate $\mathbf{A}$ occurs from the less hindered $\alpha$ face, as depicted in Scheme 6. In contrast with related hydride reductions, ${ }^{9 \mathrm{c}}$ the alternative attack from the $\beta$-face, under stereoelectronic control, ${ }^{13}$ is hindered due to the presence of the cyclohexene ring.

In summary, starting from 4-substituted cyclohexanones, we have developed a practical route to enantiopure 6-substituted cis-decahydroquinolines, the key steps being a cyclocondensation reaction of $(R)$ phenylglycinol with a 3-substituted 6-oxocyclohexenepropionate and the subsequent stereoselective carboncarbon double bond hydrogenation of the resulting tricyclic lactam. Similar cyclocondensation reactions using $(S)$-tryptophanol provide access to more complex pentacyclic derivatives related with natural products.

Acknowledgment. Financial support from the Ministerio de Ciencia e Innovación (MICINN), Spain (Project CTQ2009-07021/BQU), and the Agència de Gestió d'Ajuts Universitaris i de Recerca (AGAUR), Generalitat de Catalunya (Grant 2009-SGR-1111) is gratefully acknowledged. Thanks are also due to the MICINN (Spain) for a fellowship to L. N.

Supporting Information Available: General experimental procedures and copies of the ${ }^{1} \mathrm{H}$ and ${ }^{13} \mathrm{C}$ spectra of compounds 3-9 and 11, and X-ray crystallographic data for compounds $\mathbf{6 b}$ and the thiolactam derived from 9a. This material is available free of charge via the Internet at http://pubs.acs.org.

\footnotetext{
12 For the conformational behavior of complex quinolizidinecontaining derivatives, see: (a) Tourwé, D.; Laus, G.; Van Binst, G. $J$. Org. Chem. 1978, 43, 322-324. (b) Tourwé, D.; Van Binst, G. Heterocycles, 1978, 9, 507-533. (c) Lounasmaa, M.; Jokela, R.; Tamminen, T. Heterocycles 1985, 23, 1367-1371. (d) Lounasmaa, M.; Hanhinen, P. Heterocycles, 1999, 51, 2227-2254.

13 Deslongchamps, P. In Stereoelectronic Effects in Organic Chemistry; Baldwin, J. E., Ed.; Pergamon: Oxford, UK, 1983.
} 